\title{
Analysis of rubella disease model with non-local and non-singular fractional derivatives
}

\author{
İlknur Koca* \\ Department of Mathematics, Faculty of Sciences, Mehmet Akif Ersoy University, 15100, Burdur, Turkey \\ ikoca@mehmetakif.edu.tr
}

\section{ARTICLE INFO}

Article History:

Received 14 August 2017

Accepted 02 October 2017

Available 18 October 2017

Keywords:

Rubella disease model

Special solution

Fixed point theorem

Numerical simulations

AMS Classification 2010

34A34, 47H10, 65LO7

\section{ABSTRACT}

In this paper we investigate a possible applicability of the newly established fractional differentiation in the field of epidemiology. To do this we extend the model describing the Rubella spread by changing the derivative with the time fractional derivative for the inclusion of memory. Detailed analysis of existence and uniqueness of exact solution is presented using the Banach fixed point theorem. Finally some numerical simulations are showed to underpin the effectiveness of the used derivative.

\section{Introduction}

The aim of mathematical biology is to develop mathematical equations and to describe some physical problems encountered in biology. Noting that, the establishment of such mathematical formula is achieved using the concept of differentiation or more practically the notion of derivatives. There exist two classes of differentiation in the literatures. The first one is based on the concept of rate of change [8-11,21]. The second one is based on the convolution of some functions including exponential decay law and the generalized MittagLeffler law. The derivatives based on exponential appear naturally in many problems in nature as being able to describe the effect of fading memory. This class of derivative has been applied in several research papers for instance $[5,7,13,15,16,18$ $20,22]$. However, it was noted by several experts in the field that, this new derivative does not have a non-local kernel as its corresponding integral is not fractional, thus a new kernel was suggested by Atangana and Baleanu [6] where after some manipulations, the exponential decay kernel was replaced by the generalized Mittag-Leffler kernel. This last derivative, therefore appears to be a very powerful mathematical tools form modeling real world problems as the generalized Mittag-Leffler function is combination of the power law and exponential decay law.

Several research papers have been published using this new concept of fractional differentiation with Mittag-Leffler. More importantly the results obtained in $[1-4,6]$ revealed that, the new concept of more adequate for modeling real world problems to take into account the non-locality and also to have a memory effect. We shall note that the choice of a kernel is very important when modeling real world problems. When looking at experimental data obtained from real world observations, we can see that, many biological problems may not always follow the power law based on the function $x^{-\alpha}$ which is the kernel mostly used in the literature nowadays. For instance the case of Rubella, which is also known as the German measles or more precisely the three-day measles is enveloped and has a single-stranded RNA genome. The virus spreads via breathing

*Corresponding Author 
route and photocopies in the nasopharynx and lymph nodes. This virus can only be detected in the stream blood after a period of between 5 to 7 days when the infection has taken place, later spreads throughout the body. With its properties of teratogenic and the ability of overpass the placenta and infecting the fetus where it stops cells developing or destroys them. Such a complex dynamic will be suitable to portray a more advance concept of power with of course a non-local concept which is the property inherited by the newly established derivative with fractional order called Atangana-Baleanu derivatives [6]. This paper is therefore devoted to the analysis of the dynamic of the spread of Rubella virus exploring the Atangana-Baleanu fractional derivative. The aim of the research in this field, requires the use of the new fractional derivative for Rubella disease virus. The exactness and uniqueness of the solution of the fractional model is proved by applying the fixed-point theorem.

The remainder part of this paper is broken into sections. In Section 2, we give the definitions of the new fractional derivative with non-singular and non-local kernel. Section 3 deals with the existence of solutions for the spread of rubella disease model via Picard-Lindelof method. In Section 4 , we provide a special solution of the model which is considered using Atangala-Balenau derivative in Caputo sense. Finally in Section 5, some numerical results obtained at different instances of fractional order are presented to justify the suitability of the adopted derivative.

\section{New fractional derivative with non-singular and non-local kernel}

Let us remind the definitions of the new fractional derivative with non-singular and non-local kernel [6].

Definition 1. Let $f \in H^{1}(a, b), b>a, \alpha \in$ $[0,1]$ then, the definition of the new fractional derivative (Atangana-Baleanu derivative in Caputo sense) is given as:

$$
{ }_{a}^{A B C} D_{t}^{\alpha}(f(t))=\frac{B(\alpha)}{1-\alpha} \int_{a}^{t} f^{\prime}(x) E_{\alpha}\left[-\alpha \frac{(t-x)^{\alpha}}{1-\alpha}\right] d x
$$

where ${ }_{a}^{A B C} D_{t}^{\alpha}$ is fractional operator with MittagLeffler kernel in the Caputo sense with order $\alpha$ with respect to $t$ and $B(\alpha)=B(0)=B(1)=1$ is a normalization function [12].

Definition 2. Let $f \in H^{1}(a, b), b>a, \alpha \in$ $[0,1]$ and not differentiable then, the definition of the new fractional derivative (Atangana-Baleanu fractional derivative in Riemann-Liouville sense) is given as:

$$
{ }_{a}^{A B R} D_{t}^{\alpha}(f(t))=\frac{B(\alpha)}{1-\alpha} \frac{d}{d t} \int_{a}^{t} f(x) E_{\alpha}\left[-\alpha \frac{(t-x)^{\alpha}}{1-\alpha}\right] d x .
$$

Definition 3. The fractional integral of order $\alpha$ of a new fractional derivative is defined as:

$$
\begin{array}{r}
{ }_{a}^{A B} I_{t}^{\alpha}\{f(t)\}=\frac{1-\alpha}{B(\alpha)} f(t) \\
+\frac{\alpha}{B(\alpha) \Gamma(\alpha)} \int_{a}^{t} f(y)(t-y)^{\alpha-1} d y .
\end{array}
$$

When $\alpha$ is zero, initial function is obtained and when $\alpha$ is 1 , the ordinary integral is obtained.

\section{Existence of solutions for the spread of rubella disease model}

Let us consider the following model employing the Atangana-Baleanu fractional derivative in Caputo sense :

$$
\begin{aligned}
& { }_{0}^{A B C} D_{t}^{\alpha} S(t)=B(a)-[\lambda(a, t)+P(a)+\mu(a)] S(t), \\
& { }_{0}^{A B C} D_{t}^{\alpha} E(t)=\lambda(a, t) S(t)-(\sigma+\mu(a)) E(t) \\
& { }_{0}^{A B C} D_{t}^{\alpha} I(t)=\sigma E(t)-(\beta+\mu(a)) I(t) \\
& { }_{0}^{A B C} D_{t}^{\alpha} R(t)=\beta I(t)-\mu(a) R(t) \\
& { }_{0}^{A B C} D_{t}^{\alpha} V(t)=D(a) S(t)-\mu(a) V(t)
\end{aligned}
$$

where $S(t), E(t), I(t), R(t), V(t)$ are susceptible, latent, infectious, recovered and vaccinated parameters respectively. $P(a)$ is a parameter for which immunized by vaccination and $\lambda(a, t)$ is the force of infection of age $a$ at time $t$. Finally, $\sigma$ is the latent rate and $\beta$ is the infection rate [14]. The aim of this section is to find existence of solutions for rubella disease model with Atangana-Balenau fractional derivative. The system state is made up with $S, E, I, R, V$. The above system (4) can be converted to Volterra type integral equation with the Atangana-Baleanu fractional integral.

Theorem 1. The following time fractional ordinary differential equation

$$
{ }_{0}^{A B C} D_{t}^{\alpha}(f(t))=u(t)
$$


has a unique solution with taking the inverse Laplace transform and using the convolution theorem below [4]:

$f(t)=\frac{1-\alpha}{B(\alpha)} u(t)+\frac{\alpha}{B(\alpha) \Gamma(\alpha)} \int_{a}^{t} u(y)(t-y)^{\alpha-1} d y$.

By the theorem above, the model can be written as (7):

$$
\left\{\begin{array}{l}
S(t)-g_{1}(t)=\frac{1-\alpha}{B(\alpha)}\{B(a)-[\lambda(a, t)+P(a)+\mu(a)] S(t)\} \\
+\frac{\alpha}{B(\alpha) \Gamma(\alpha)} \int_{0}^{t}(t-y)^{\alpha-1} \\
\times\{B(a)-[\lambda(a, y)+P(a)+\mu(a)] S(y)\} d y, \\
E(t)-g_{2}(t)=\frac{1-\alpha}{B(\alpha)}\{\lambda(a, t) S(t)-(\sigma+\mu(a)) E(t)\} \\
+\frac{\alpha}{B(\alpha) \Gamma(\alpha)} \int_{0}^{t}(t-y)^{\alpha-1} \\
\times\{\lambda(a, y) S(y)-(\sigma+\mu(a)) E(y)\} d y, \\
I(t)-g_{3}(t)=\frac{1-\alpha}{B(\alpha)}\{\sigma E(t)-(\beta+\mu(a)) I(t)\} \\
+\frac{\alpha}{B(\alpha) \Gamma(\alpha)} \int_{0}^{t}(t-y)^{\alpha-1}\{\sigma E(y)-(\beta+\mu(a)) I(y)\} d y, \\
R(t)-g_{4}(t)=\frac{1-\alpha}{B(\alpha)}\{\beta I(t)-\mu(a) R(t)\} \\
+\frac{\alpha}{B(\alpha) \Gamma(\alpha)} \int_{0}^{t}(t-y)^{\alpha-1}\{\beta I(y)-\mu(a) R(y)\} d y, \\
V(t)-g_{5}(t)=\frac{1-\alpha}{B(\alpha)}\{D(a) S(t)-\mu(a) V(t)\} \\
+\frac{\alpha}{B(\alpha) \Gamma(\alpha)} \int_{0}^{t}(t-y)^{\alpha-1}\{D(a) S(y)-\mu(a) V(y)\} d y,
\end{array}\right.
$$

The above system (7) of equations can be iteratively represented as:

$$
\begin{aligned}
& \left\{\begin{array}{c}
S_{0}(t)=g_{1}(t), \\
E_{0}(t)=g_{2}(t), \\
I_{0}(t)=g_{3}(t), \\
R_{0}(t)=g_{4}(t), \\
V_{0}(t)=g_{5}(t) .
\end{array}\right. \\
& S_{n+1}(t)=\frac{1-\alpha}{B(\alpha)} \\
& \times\left\{B(a)-[\lambda(a, t)+P(a)+\mu(a)] S_{n}(t)\right\} \\
& +\frac{\alpha}{B(\alpha) \Gamma(\alpha)} \int_{0}^{t}(t-y)^{\alpha-1} \\
& \times\left\{B(a)-[\lambda(a, y)+P(a)+\mu(a)] S_{n}(y)\right\} d y, \\
& E_{n+1}(t)=\frac{1-\alpha}{B(\alpha)}\left\{\lambda(a, t) S_{n}(t)-(\sigma+\mu(a)) E_{n}(t)\right\} \\
& +\frac{\alpha}{B(\alpha) \Gamma(\alpha)} \int_{0}^{t}(t-y)^{\alpha-1} \\
& \times\left\{\lambda(a, y) S_{n}(y)-(\sigma+\mu(a)) E_{n}(y)\right\} d y,
\end{aligned}
$$

$$
\begin{aligned}
I_{n+1}(t)= & \frac{1-\alpha}{B(\alpha)}\left\{\sigma E_{n}(t)-(\beta+\mu(a)) I_{n}(t)\right\} \\
+ & \frac{\alpha}{B(\alpha) \Gamma(\alpha)} \int_{0}^{t}(t-y)^{\alpha-1} \\
\times & \left\{\sigma E_{n}(y)-(\beta+\mu(a)) I_{n}(y)\right\} d y, \\
R_{n+1}(t)= & \frac{1-\alpha}{B(\alpha)}\left\{\beta I_{n}(t)-\mu(a) R_{n}(t)\right\} \\
& +\frac{\alpha}{B(\alpha) \Gamma(\alpha)} \int_{0}^{t}(t-y)^{\alpha-1} \\
& \times\left\{\beta I_{n}(y)-\mu(a) R_{n}(y)\right\} d y, \\
V_{n+1}(t)= & \frac{1-\alpha}{B(\alpha)}\left\{D(a) S_{n}(t)-\mu(a) V_{n}(t)\right\} \\
+ & \frac{\alpha}{B(\alpha) \Gamma(\alpha)} \int_{0}^{t}(t-y)^{\alpha-1} \\
& \times\left\{D(a) S_{n}(y)-\mu(a) V_{n}(y)\right\} d y .
\end{aligned}
$$

As the exact solution of the iterative formula of a Picard series used here converges toward the exact solution as the number of series terms tends to infinity. If we take the limit with greater than $n$, we expect to obtain the exact solution of equation as below:

$$
\left\{\begin{aligned}
\lim _{n \rightarrow \infty} S_{n}(t) & =S(t), \\
\lim _{n \rightarrow \infty} E_{n}(t) & =E(t), \\
\lim _{n \rightarrow \infty} I_{n}(t) & =I(t), \\
\lim _{n \rightarrow \infty} R_{n}(t) & =R(t), \\
\lim _{n \rightarrow \infty} V_{n}(t) & =V(t) .
\end{aligned}\right.
$$

\subsection{Existence of solution via Picard-Lindelof method}

Let us define the following operator for showing the existence of solution:

$$
\begin{aligned}
& f_{1}(a, t)=B(a)-[\lambda(a, t)+P(a)+\mu(a)] S(t), \\
& f_{2}(a, t)=\lambda(a, t) S(t)-(\sigma+\mu(a)) E(t), \\
& f_{3}(a, t)=\sigma E(t)-(\beta+\mu(a)) I(t) \\
& f_{4}(a, t)=\beta I(t)-\mu(a) R(t) \\
& f_{5}(a, t)=D(a) S(t)-\mu(a) V(t) .
\end{aligned}
$$

Let

$$
\begin{aligned}
& N_{1}=\sup _{C\left[b, c_{1}\right]}\left\|f_{1}(a, t)\right\|, N_{2}=\sup _{C\left[b, c_{2}\right]}\left\|f_{2}(a, y)\right\|, \\
& N_{3}=\sup _{C\left[b, c_{3}\right]}\left\|f_{3}(a, z)\right\|, N_{4}=\sup _{C\left[b, c_{4}\right]}\left\|f_{4}(a, p)\right\|, \\
& N_{5}=\sup _{C\left[b, c_{5}\right]}\left\|f_{5}(a, r)\right\|,
\end{aligned}
$$


where

$$
\begin{aligned}
& C\left[b, c_{1}\right]=[t-b, t+b] \times\left[x-c_{1}, x+c_{1}\right]=B_{1} \times C_{1}, \\
& C\left[b, c_{2}\right]=[t-b, t+b] \times\left[x-c_{2}, x+c_{2}\right]=B_{1} \times C_{2}, \\
& C\left[b, c_{3}\right]=[t-b, t+b] \times\left[x-c_{3}, x+c_{3}\right]=B_{1} \times C_{3}, \\
& C\left[b, c_{4}\right]=[t-b, t+b] \times\left[x-c_{4}, x+c_{4}\right]=B_{1} \times C_{4}, \\
& C\left[b, c_{5}\right]=[t-b, t+b] \times\left[x-c_{5}, x+c_{5}\right]=B_{1} \times C_{5} .
\end{aligned}
$$

We will make use of Banach fixed-point theorem using the metric on $C\left[b, c_{i}\right],(i=1,2, \ldots, 5)$ made by the uniform norm

$$
\|X(t)\|_{\infty}=\sup _{t \in[t-b, t+b]}|f(t)|
$$

The next operator is defined between the two functional spaces of continuous functions, $\mathrm{Pi}$ card's operator as follows:

$$
\begin{aligned}
& O: C\left(B_{1}, C_{1}, C_{2}, C_{3}, C_{4}, C_{5}\right) \\
& \rightarrow C\left(B_{1}, C_{1}, C_{2}, C_{3}, C_{4}, C_{5}\right) .
\end{aligned}
$$

For simplicity, let us define $f_{i}(a, t)=X(t)$, $f_{i}(a, 0)=X_{0}(t),(i=1,2, \ldots, 5)$. Then the system is reduced the following:

$$
\begin{aligned}
O X(t) & =X_{0}(t)+F(t, X(t)) \frac{1-\alpha}{B(\alpha)} \\
& +\frac{\alpha}{B(\alpha) \Gamma(\alpha)} \int_{0}^{t}(t-y)^{\alpha-1} F(y, X(y)) d y
\end{aligned}
$$

where $X$ is the matrice of given as

$$
\begin{aligned}
& X(t)=\left\{\begin{array}{l}
S(t) \\
E(t) \\
I(t) \\
R(t) \\
V(t)
\end{array}, X_{0}(t)=\left\{\begin{array}{l}
S(0) \\
E(0) \\
I(0) \\
R(0) \\
V(0)
\end{array}\right.\right. \\
& F(a, X(t))=\left\{\begin{array}{l}
f_{1}(a, t) \\
f_{2}(a, t) \\
f_{3}(a, t) \\
f_{4}(a, t) \\
f_{5}(a, t)
\end{array}\right.
\end{aligned}
$$

Let us assume that the physical problem under investigation satisfies followings:

$$
\|X(t)\|_{\infty} \leq \max \left\{c_{1}, c_{2}, c_{3}, c_{4}, c_{5}\right\}
$$

$$
\begin{aligned}
& \left\|O X(t)-X_{0}(t)\right\| \\
& =\left\|F(t, X(t)) \frac{1-\alpha}{B(\alpha)}+\frac{\alpha}{B(\alpha) \Gamma(\alpha)} \int_{0}^{t}(t-y)^{\alpha-1} F(y, X(y)) d y\right\| \\
& \leq \frac{1-\alpha}{B(\alpha)}\|F(t, X(t))\| \\
& +\frac{\alpha}{B(\alpha) \Gamma(\alpha)} \int_{0}^{t}(t-y)^{\alpha-1}\|F(y, X(y))\| d y \\
& \leq \frac{1-\alpha}{B(\alpha)} N=\max \left\{N_{1}, N_{2}, N_{3}, N_{4}, N_{5}\right\} \\
& +\frac{\alpha}{B(\alpha)} N b^{\alpha}<b N \leq c=\max \left\{c_{1}, c_{2}, c_{3}, c_{4}, c_{5}\right\}
\end{aligned}
$$

where we demand that

$$
b<\frac{c}{N}
$$

Also we evaluate the following equality

$$
\left\|O X_{1}-O X_{2}\right\|_{\infty}=\sup _{t \in B}\left|X_{1}-X_{2}\right| .
$$

Nonetheless using the definition of our defined operator, we have

$$
\left\|O X_{1}-O X_{2}\right\|
$$

$$
\begin{aligned}
& =\left\|\begin{array}{c}
\left\{F\left(t, X_{1}(t)\right)-F\left(t, X_{2}(t)\right)\right\} \frac{1-\alpha}{B(\alpha)} \\
+\frac{\alpha}{B(\alpha) \Gamma(\alpha)} \int_{0}^{t}(t-l)^{\alpha-1}\left\{\begin{array}{c}
F\left(l, X_{1}(l)\right) \\
-F\left(l, X_{2}(l)\right)
\end{array}\right\} d l \|
\end{array}\right\| \\
& \leq \frac{1-\alpha}{B(\alpha)}\left\|F\left(t, X_{1}(t)\right)-F\left(t, X_{2}(t)\right)\right\| \\
& +\frac{\alpha}{B(\alpha) \Gamma(\alpha)} \int_{0}^{t}(t-y)^{\alpha-1} \\
& \times\left\|F\left(l, X_{1}(y)\right)-F\left(l, X_{2}(y)\right)\right\| d y \\
& \leq \frac{1-\alpha}{B(\alpha)} q\left\|X_{1}(t)-X_{2}(t)\right\| \\
& +\frac{\alpha q}{B(\alpha) \Gamma(\alpha)} \int_{0}^{t}(t-y)^{\alpha-1}\left\|X_{1}(y)-X_{2}(y)\right\| d y \\
& \leq\left\{\frac{1-\alpha}{B(\alpha)} q+\frac{\alpha q b^{\alpha}}{B(\alpha) \Gamma(\alpha)}\right\}\left\|X_{1}(t)-X_{2}(t)\right\| \\
& \leq b q\left\|X_{1}(t)-X_{2}(t)\right\| .
\end{aligned}
$$

with $q<1$ since $F$ is a contraction we have that $b q<1$, thus the defined operator $O$ is a contraction. So system has a unique set of solution.

\section{Special solutions via iteration approach}

The aim of this section is to provide a special solution of the model which is considered using 
Atangala-Balenau derivative in Caputo sense. Let us apply the Sumudu transform on both sides of equation (4) together with an iterative method. We shall give the Sumudu transform of AtanganaBalenau fractional derivative in Caputo sense below:

Theorem 2. Let $f \in H^{1}(a, b), b>a, \alpha \in[0,1]$ then, the Sumudu transform of Atangana-Balenau fractional derivative in Caputo sense is given as:

$$
\begin{aligned}
& S T\left\{\begin{array}{l}
{ }_{0}^{A B C} \\
0
\end{array} D_{t}^{\alpha}(f(t))\right\} \\
& =\frac{B(\alpha)}{1-\alpha}\left(\alpha \Gamma(\alpha+1) E_{\alpha}\left(-\frac{1}{1-\alpha} p^{\alpha}\right)\right) \\
& \times(S T(f(t))-f(0)) .
\end{aligned}
$$

Proof. Proof of the theorem can be found in [4].

To solve Equation (4), we apply the Sumudu transform of the Atangana-Balenau fractional derivative of $f(t)$ on system with both sides. Then we obtain below:

$$
\begin{aligned}
& \frac{B(\alpha)}{1-\alpha}\left(\alpha \Gamma(\alpha+1) E_{\alpha}\left(-\frac{1}{1-\alpha} p^{\alpha}\right)\right)(S T(S(t))-S(0)) \\
& =S T\{B(a)-[\lambda(a, t)+P(a)+\mu(a)] S(t)\}, \\
& \frac{B(\alpha)}{1-\alpha}\left(\alpha \Gamma(\alpha+1) E_{\alpha}\left(-\frac{1}{1-\alpha} p^{\alpha}\right)\right)(S T(E(t))-E(0)) \\
& =S T\{\lambda(a, t) S(t)-(\sigma+\mu(a)) E(t)\}, \\
& \frac{B(\alpha)}{1-\alpha}\left(\alpha \Gamma(\alpha+1) E_{\alpha}\left(-\frac{1}{1-\alpha} p^{\alpha}\right)\right)(S T(I(t))-I(0)) \\
& =S T\{\sigma E(t)-(\beta+\mu(a)) I(t)\}, \\
& \frac{B(\alpha)}{1-\alpha}\left(\alpha \Gamma(\alpha+1) E_{\alpha}\left(-\frac{1}{1-\alpha} p^{\alpha}\right)\right)(S T(R(t))-R(0)) \\
& =S T\{\beta I(t)-\mu(a) R(t)\}, \\
& \frac{B(\alpha)}{1-\alpha}\left(\alpha \Gamma(\alpha+1) E_{\alpha}\left(-\frac{1}{1-\alpha} p^{\alpha}\right)\right)(S T(V(t))-V(0)) \\
& =S T\{D(a) S(t)-\mu(a) V(t)\} .
\end{aligned}
$$

Rearranging, we obtain following inequalities where,

$$
\begin{aligned}
& S T(S(t))=S(0) \\
& +\theta * S T\{B(a)-[\lambda(a, t)+P(a)+\mu(a)] S(t)\},
\end{aligned}
$$

$$
\begin{aligned}
& S T(E(t))=E(0) \\
& +\theta * S T\{\lambda(a, t) S(t)-(\sigma+\mu(a)) E(t)\}, \\
& S T(I(t))=I(0) \\
& +\theta * S T\{\sigma E(t)-(\beta+\mu(a)) I(t)\}, \\
& S T(R(t))=R(0) \\
& +\theta * S T\{\beta I(t)-\mu(a) R(t)\}, \\
& S T(V(t))=V(0) \\
& +\theta * S T\{D(a) S(t)-\mu(a) V(t)\} .
\end{aligned}
$$

For simplicity, here

$$
\theta=\frac{1-\alpha}{B(\alpha)\left(\alpha \Gamma(\alpha+1) E_{\alpha}\left(-\frac{1}{1-\alpha} p^{\alpha}\right)\right)},
$$

is considered and "*" means multiplication sign . We next obtain the following recursive formula;

$$
\begin{gathered}
S_{n+1}(t)=S_{n}(0) \\
+S T^{-1}\left\{\theta * S T\left\{B(a)-[\lambda(a, t)+P(a)+\mu(a)] S_{n}(t)\right\}\right\}, \\
E_{n+1}(t)=E_{n}(0) \\
+S T^{-1}\left\{\theta * S T\left\{\lambda(a, t) S_{n}(t)-(\sigma+\mu(a)) E_{n}(t)\right\}\right\}, \\
I_{n+1}(t)=I_{n}(0) \\
+S T^{-1}\left\{\theta * S T\left\{\sigma E_{n}(t)-(\beta+\mu(a)) I_{n}(t)\right\}\right\}, \\
R_{n+1}(t)=R_{n}(0) \\
+S T^{-1}\left\{\theta * S T\left\{\beta I_{n}(t)-\mu(a) R_{n}(t)\right\}\right\}, \\
V_{n+1}(t)=V_{n}(0) \\
+S T^{-1}\left\{\theta * S T\left\{D(a) S_{n}(t)-\mu(a) V_{n}(t)\right\}\right\} .
\end{gathered}
$$

Therefore, the solution of equation (24) approximate to following

$$
\begin{aligned}
S(t) & =\lim _{n \rightarrow \infty} S_{n}(t), \\
E(t) & =\lim _{n \rightarrow \infty} E_{n}(t), \\
I(t) & =\lim _{n \rightarrow \infty} I_{n}(t), \\
R(t) & =\lim _{n \rightarrow \infty} R_{n}(t), \\
V(t) & =\lim _{n \rightarrow \infty} V_{n}(t) .
\end{aligned}
$$

\subsection{Application of fixed-point theorem for stability analysis of iteration method}

Let $(X,\|\|$.$) be a Banach space and H$ a self-map of $X$. Let $y_{n+1}=g\left(H, y_{n}\right)$ be recurcive procedure. Suppose that, $F(H)$ the fixed-point set of 
$H$ has at least one element and that $y_{n}$ converges to a point $p \in F(H)$. Let $\left\{x_{n}\right\} \subseteq X$ and define $e_{n}=\left\|x_{n+1}-g\left(H, x_{n}\right)\right\|$. If $\lim _{n \rightarrow \infty} e_{n}=0$ implies that $\lim _{n \rightarrow \infty} x_{n}=p$, then the iteration method $y_{n+1}=g\left(H, y_{n}\right)$ is $H$-Stable. Then let we assume that, our sequence $\left\{x_{n}\right\}$ has an upper boundary . If all these conditions are satisfied for $y_{n+1}=H y_{n}$ which is known as Picard's iteration, consequently the iteration is $H$-Stable. We shall then state the following theorem.

Theorem 3. Let $(X,\|\|$.$) be a Banach space and$ $H$ a self-map of $X$ satisfying

$$
\left\|H_{x}-H_{y}\right\| \leq K\left\|x-H_{x}\right\|+k\|x-y\|,
$$

for all $x, y$ in $X$ where $0 \leq K, 0 \leq k<1$. Suppose that $H$ is Picard $H$-Stable [17].

Let us consider the following recursive formula equation (27) with (4) where

$$
\theta=\frac{1-\alpha}{B(\alpha)\left(\alpha \Gamma(\alpha+1) E_{\alpha}\left(-\frac{1}{1-\alpha} p^{\alpha}\right)\right)},
$$

is the fractional Lagrange multiplier.

Theorem 4. Let $H$ be a self-map defined as (27) as below.

$$
\begin{gathered}
H\left(S_{n}(t)\right)=S_{n+1}(t)=S_{n}(t) \quad(27) \\
+S T^{-1}\left\{\theta * S T\left\{B(a)-[\lambda(a, t)+P(a)+\mu(a)] S_{n}(t)\right\}\right\}, \\
H\left(E_{n}(t)\right)=E_{n+1}(t)=E_{n}(t) \\
+S T^{-1}\left\{\theta * S T\left\{\lambda(a, t) S_{n}(t)-(\sigma+\mu(a)) E_{n}(t)\right\}\right\}, \\
H\left(I_{n}(t)\right)=I_{n+1}(t)=I_{n}(t) \\
+S T^{-1}\left\{\theta * S T\left\{\sigma E_{n}(t)-(\beta+\mu(a)) I_{n}(t)\right\}\right\}, \\
H\left(R_{n}(t)\right)=R_{n+1}(t)=R_{n}(t) \\
+S T^{-1}\left\{\theta * S T\left\{\beta I_{n}(t)-\mu(a) R_{n}(t)\right\}\right\}, \\
H\left(V_{n}(t)\right)=V_{n+1}(t)=V_{n}(t) \\
+S T^{-1}\left\{\theta * S T\left\{D(a) S_{n}(t)-\mu(a) V_{n}(t)\right\}\right\} .
\end{gathered}
$$

Then (27) is $H$-stable in $L^{1}(a, b)$ if following statement can be obtained.

$$
\begin{aligned}
(1-[\lambda(a, t)+P(a)+\mu(a)] A(\gamma)) & <1, \\
(1+\lambda(a, t) B(\gamma)-(\sigma+\mu(a)) C(\gamma)) & <1, \\
(1+\sigma D(\gamma)-(\beta+\mu(a)) E(\gamma)) & <1, \\
(1+\beta F(\gamma)-\mu(a) G(\gamma)) & <1, \\
(1+D(a) H(\gamma)-\mu(a) J(\gamma)) & <1 .
\end{aligned}
$$

Proof. Let we start with showing that $H$ has a fixed point. To achieve this, we evaluate the followings for all $(n, m) \in \mathbb{N} \times \mathbb{N}$.

$$
\begin{gathered}
H\left(S_{n}(t)\right)-H\left(S_{m}(t)\right)=S_{n}(t)-S_{m}(t) \\
+S T^{-1}\left\{\theta * S T\left\{B(a)-[\lambda(a, t)+P(a)+\mu(a)] S_{n}(t)\right\}\right\} \\
-S T^{-1}\left\{\theta * S T\left\{B(a)-[\lambda(a, t)+P(a)+\mu(a)] S_{m}(t)\right\}\right\} .
\end{gathered}
$$

Let us consider (29) and apply norm on both sides and without loss of generality

$$
\begin{gathered}
\left\|H\left(S_{n}(t)\right)-H\left(S_{m}(t)\right)\right\| \\
=\left\|+S T^{-1}\left\{\theta * S T\left\{\begin{array}{c}
S_{n}(t)-S_{m}(t) \\
B(a)-\lambda(a, t)+P(a)+\mu(a)] S_{n}(t) \\
-\left(B(a)-[\lambda(a, t)+P(a)+\mu(a)] S_{m}(t)\right)
\end{array}\right\}\right\}\right\| \\
\leq\left\|S_{n}(t)-S_{m}(t)\right\|
\end{gathered}
$$

Now we obtain :

$$
\begin{aligned}
& \left\|H\left(S_{n}(t)\right)-H\left(S_{m}(t)\right)\right\| \leq\left\|S_{n}(t)-S_{m}(t)\right\| \\
& \times(1-[\lambda(a, t)+P(a)+\mu(a)] A(\gamma)),
\end{aligned}
$$

where $A(\gamma)$ is the $S T^{-1}\{\theta * S T\}$. Since all solutions have same role also we have following:

$$
\begin{aligned}
& \left\|H\left(E_{n}(t)\right)-H\left(E_{m}(t)\right)\right\| \leq\left\|E_{n}(t)-E_{m}(t)\right\| \\
& \times(1+\lambda(a, t) B(\gamma)-(\sigma+\mu(a)) C(\gamma)), \\
& \left\|H\left(I_{n}(t)\right)-H\left(I_{m}(t)\right)\right\| \leq\left\|I_{n}(t)-I_{m}(t)\right\| \\
& \times(1+\sigma D(\gamma)-(\beta+\mu(a)) E(\gamma)), \\
& \left\|H\left(R_{n}(t)\right)-H\left(R_{m}(t)\right)\right\| \leq\left\|R_{n}(t)-R_{m}(t)\right\| \\
& \times(1+\beta F(\gamma)-\mu(a) G(\gamma)), \\
& \left\|H\left(V_{n}(t)\right)-H\left(V_{m}(t)\right)\right\| \leq\left\|V_{n}(t)-V_{m}(t)\right\| \\
& \times(1+D(a) H(\gamma)-\mu(a) J(\gamma)) .
\end{aligned}
$$

For

$$
\begin{aligned}
(1-[\lambda(a, t)+P(a)+\mu(a)] A(\gamma)) & <1, \\
(1+\lambda(a, t) B(\gamma)-(\sigma+\mu(a)) C(\gamma)) & <1, \\
(1+\sigma D(\gamma)-(\beta+\mu(a)) E(\gamma)) & <1, \\
(1+\beta F(\gamma)-\mu(a) G(\gamma)) & <1, \\
(1+D(a) H(\gamma)-\mu(a) J(\gamma)) & <1,
\end{aligned}
$$

then H-self mapping has a fixed point. Also nonlinear mapping $\mathrm{H}$ has to satisfy the conditions. So let we assume

$$
k=\left\{\begin{array}{c}
K=(0,0,0,0,0) \\
(1-[\lambda(a, t)+P(a)+\mu(a)] A(\gamma)) \\
(1+\lambda(a, t) B(\gamma)-(\sigma+\mu(a)) C(\gamma)) \\
(1+\sigma D(\gamma)-(\beta+\mu(a)) E(\gamma)) \\
(1+\beta F(\gamma)-\mu(a) G(\gamma)) \\
(1+D(a) H(\gamma)-\mu(a) J(\gamma))
\end{array},\right.
$$


then all conditions of Theorem 3 hold. This completes the proof.

\section{Numerical Simulation}

In this part, we present the numerical replication of the model for different values of fractional order using the proposed numerical scheme. The numerical simulations are shown in figure $1,2,3$, and 4. Figures 1 is considered alpha to be 0.95 , figure 2 is considered alpha to be 0.65 , figure 3 is considered alpha to be 0.45 and finally in figure 4 is considered alpha to be 0.05 . The paremeters used in this simulations are given below:

$$
\begin{aligned}
& B=100, \quad P=0.3, \quad \lambda=0.4, \\
& \mu=0.4, \quad \sigma=0.3, \quad \beta=0.4
\end{aligned} .
$$

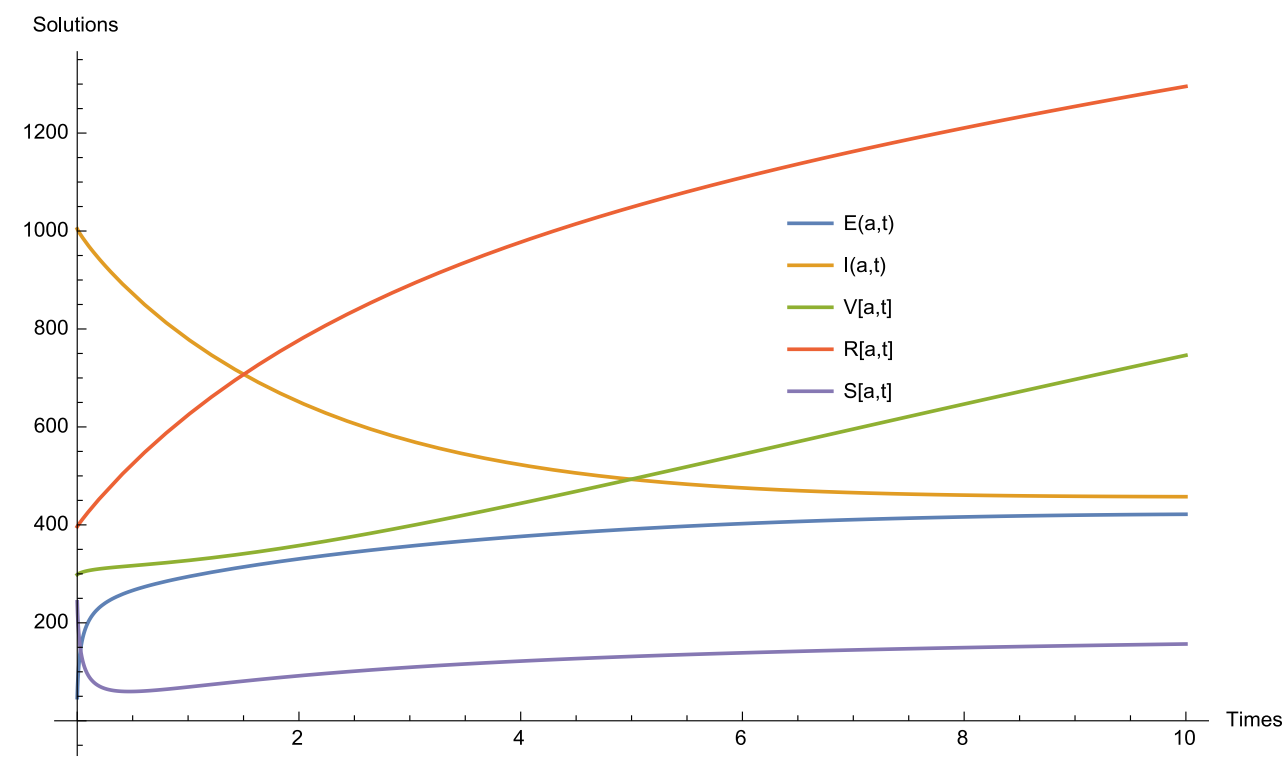

$n$

Figure 1 : Numerical simulation of solution for $\alpha=0.95$

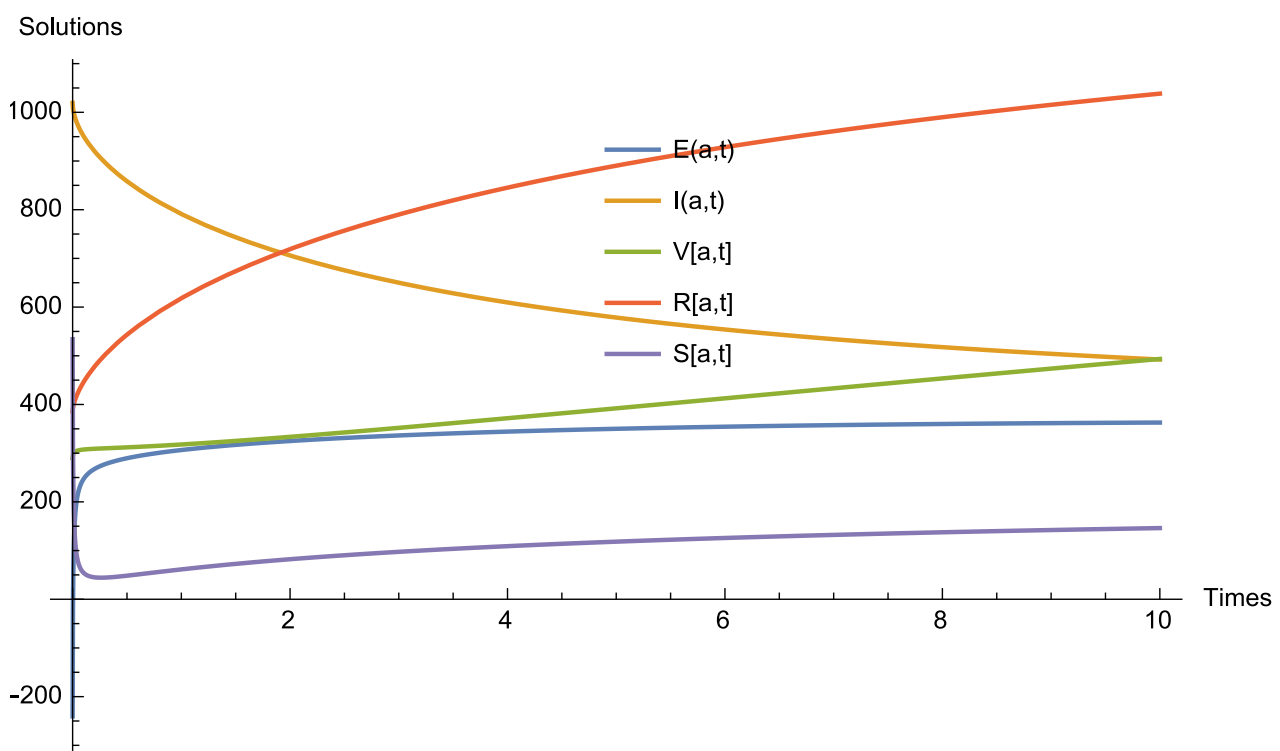

Figure 2: Numerical simulation of solution for $\alpha=0.65$ 


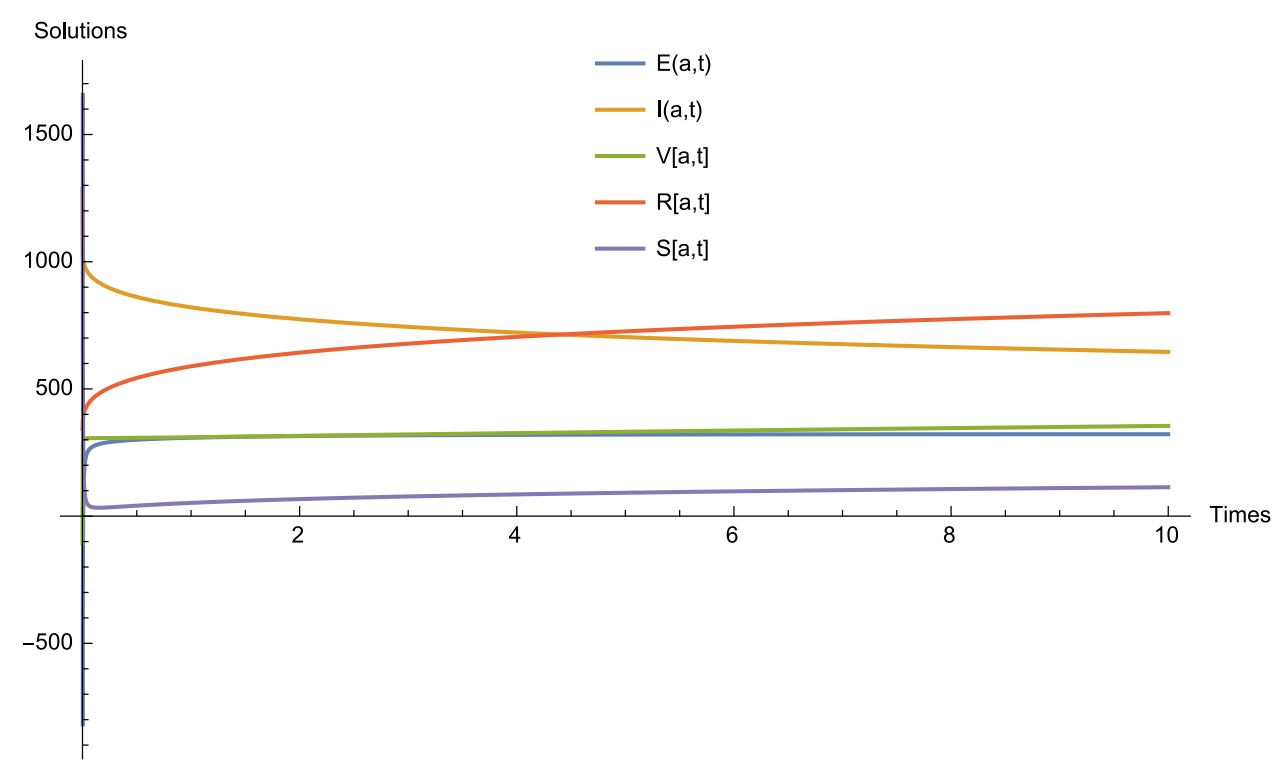

Figure 3 : Numerical simulation of solution for $\alpha=0.45$

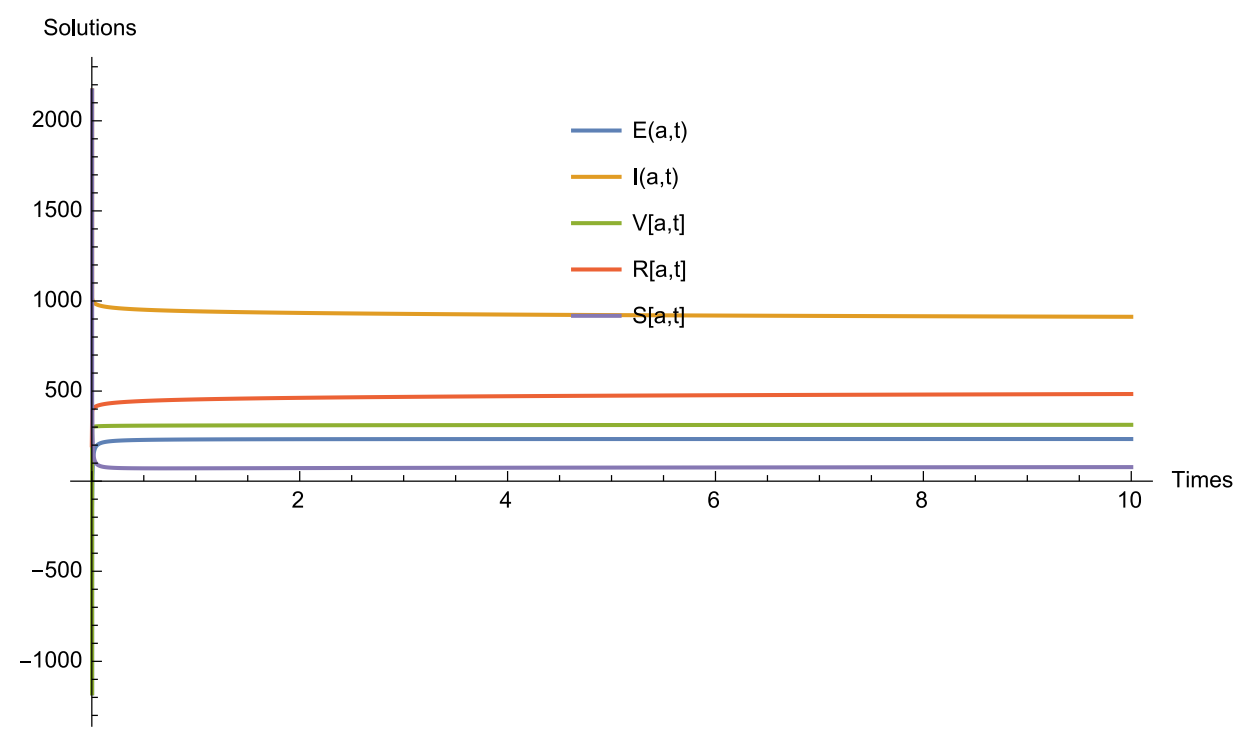

Figure 4 : Numerical simulation of solution for $\alpha=0.05$

\section{Conclusion}

In this work, we have extended the model of rubella disease to the concept of fractional differential based on the Mittag-Leffler. We studied the existence of the generalized model using the fixed-point theorem. We presented the derivation of the solution using the Sumudu transform of Atanagana-Balenau derivative in Caputo sense. The stability analysis of the method is validated with the $H$-stable approach. Finally. Numerical simulations presented for different values of $\alpha$.

\section{References}

[1] Abdeljawad, T. and Baleanu, D. (2016). Discrete fractional differences with nonsingular discrete MittagLeffler kernels. Advances in Difference Equations. (1), 232 .
[2] Abdeljawad, T. and Baleanu, D. (2016). Integration by parts and its applications of a new nonlocal fractional derivative with Mittag-Leffler nonsingular kernel. arXiv preprint arXiv, 1607.00262.

[3] Alkahtani, B.S.T. (2016). Chua's circuit model with Atangana-Baleanu derivative with fractional order. Chaos Solitons and Fractals. 89, 547-551.

[4] Atangana, A. and Koca, I. (2016). Chaos in a simple nonlinear system with Atangana-Baleanu derivatives with fractional order. Chaos, Solitons and Fractals. 89, 447-454.

[5] Atangana, A. and Koca, I. (2016). On the new fractional derivative and application to Nonlinear Baggs and Freedman model. Journal of Nonlinear Sciences and Applications. (9), 2467-2480.

[6] Atangana, A. and Baleanu, D. (2016). New fractional derivatives with nonlocal and non-singular kernel: theory and application to heat transfer model. Thermal Science. 20 (2), 763-769.

[7] Atangana, A. and Owolabi, K.M. (2017). New numerical approach for fractional differential equations. preprint, arXiv, 1707.08177. 
[8] Baskonus, H.M. and Bulut, H. (2015). On the numerical solutions of some fractional ordinary differential equations by fractional Adams-BashforthMoulton Method. Open Mathematics. 13 (1), 547-556.

[9] Baskonus, H.M. and Bulut, H. (2016). Regarding on the prototype solutions for the nonlinear fractionalorder biological population model. AIP Conference Proceedings. 1738, 290004.

[10] Baskonus, H.M., Mekkaoui, T., Hammouch, Z. and Bulut, H. (2015). Active control of a chaotic fractional order economic system. Entropy. 17 (8), 5771-5783.

[11] Baskonus, H.M., Hammouch, Z., Mekkaoui, T. and Bulut, H. (2016). Chaos in the fractional order logistic delay system: circuit realization and synchronization. AIP Conference Proceedings, 1738, 290005.

[12] Caputo, M. \& Fabrizio, M. (2015). A new definition of fractional derivative without singular kernel. Progress in Fractional Differentiation and Applications. 1, 7385.

[13] Coronel-Escamilla, A., Gómez-Aguilar, J.F., Baleanu, D., Escobar-Jiménez, R. F., Olivares-Peregrino, V. H., and Abundez-Pliego, A. (2016). Formulation of EulerLagrange and Hamilton equations involving fractional operators with regular kernel. Advances in Difference Equations. (1), 283.

[14] Gay, N.J., Pelletier, L. and Duclos, P. (1998). Modelling the incidence of measles in Canada: An assessment of the options for vaccination policy. Vaccine. 16, 794-801.

[15] Gencoglu, M.T., Baskonus, H.M. and Bulut, H. (2017). Numerical simulations to the nonlinear model of interpersonal Relationships with time fractional derivative. AIP Conference Proceedings. 1798, 1-9, 020103.

[16] Gómez-Aguilar, J.F., Morales-Delgado, V.F., TanecoHernández, M.A., Baleanu, D., Escobar-Jiménez,
R.F., and Al Qurashi, M.M. (2016). Analytical solutions of the electrical RLC circuit via LiouvilleCaputo operators with local and non-Local kernels. Entropy. 18 (8), 402.

[17] Odibat, Z.M. and Momani, S. (2006). Application of variational iteration method to nonlinear differential equation of fractional order. International Journal of Nonlinear Sciences and Numerical Simulations. 7, 2734.

[18] Owolabi, K.M. (2016). Numerical solution of diffusive HBV model in a fractional medium. SpringerPlus. 5, 19 pages.

[19] Owolabi, K.M. and Atangana, A. (2017). Numerical approximation of nonlinear fractional parabolic differential equations with Caputo-Fabrizio derivative in Riemann-Liouville sense. Chaos, Solitons and Fractals. 99, 171-179.

[20] Owolabi, K.M. (2017). Mathematical modelling and analysis of two-component system with Caputo fractional derivative order. Chaos Solitons and Fractals. 103, 544-554.

[21] Ozalp, N. and Koca, I. (2012). A fractional order nonlinear dynamical model of interpersonal relationships. Advances in Difference Equations. 189, 7 pages.

[22] Singh, J., Kumar, D., Al Qurashi, M. and Baleanu, D. (2017). A new fractional model for giving up smoking dynamics. Advances in Difference Equations, 88 (1), 16 pages.

Ilknur Koca received the B.Sc. and M.Sc. degrees in 2007 and 2009 from the Department of Mathematics from Ankara University, Turkey respectively. She received her Ph.D. degree from the same university in 2013. She is working as an Assistant Professor at Department of Mathematics of Mehmet Akif Ersoy University. Her research interests are methods and applications of partial and ordinary differential equations, fractional differential equations, iterative methods.

An International Journal of Optimization and Control: Theories \& Applications (http://ijocta.balikesir.edu.tr)

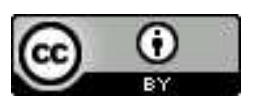

This work is licensed under a Creative Commons Attribution 4.0 International License. The authors retain ownership of the copyright for their article, but they allow anyone to download, reuse, reprint, modify, distribute, and/or copy articles in IJOCTA, so long as the original authors and source are credited. To see the complete license contents, please visit http://creativecommons.org/licenses/by/4.0/. 